\title{
Glycosphingolipides et fusion virus-cellule : données actuelles montrant le rôle des micro- domaines membranaires dans le cycle d'infection du VIH-1
}

\author{
Glycosphingolipids and virus-cell fusion: current data highlighting the role of membrane \\ microdomains in the HIV-1 infection cycle
}

Oléagineux, Corps Gras, Lipides. Volume 7, Numéro 5, 449-55, Septembre - Octobre 2000, Fondamental

Auteur(s) : Djilali HAMMACHE, Nouara YAHI, Jacques FANTINI, Institut méditerranéen de recherche en nutrition, Faculté des sciences de Saint-Jérôme, 13397 Marseille Cedex 20.

Résumé : Depuis plusieurs années, nous étudions les mécanismes moléculaires responsables de la fusion du virus de l'immunodéficience humaine $(\mathrm{VIH})$ avec la membrane plasmique des cellules cibles. Ces travaux ont permis de préciser le rôle essentiel joué par les micro-domaines de glycosphingolipides au cours de la fusion virus-cellule. En particulier, nous avons pu reconstituer un complexe de fusion fonctionnel faisant intervenir les différents partenaires moléculaires de la fusion : un micro-domaine de glycosphingolipide se présentant sous la forme d'un film monomoléculaire à l'interface eau-air, le récepteur CD4 et la glycoprotéine externe de l'enveloppe du virus, la gp120. La dynamique des interactions moléculaires dans ce complexe de fusion a pu être mesurée à l'aide d'un micro-tensiomètre. Ce système expérimental pourrait permettre d'évaluer l'activité d'inhibiteurs de fusion tels que des analogues synthétiques de glycosphingolipides.

Mots-clés : virus, VIH, sida, lipides, fusion, membrane, infection.

Summary : Since several years we have been studying the molecular mechanisms involved in the fusion between the human immunodeficiency virus (HIV) and the plasma membrane of target cells. We have contributed to demonstrate the essential role played by glycosphingolipid microdomains during the virus-cell fusion process. In particular, we have been able to reconstitute a functional fusion complex involving: i) a monomolecular film of glycosphingolipid mimicking a plasma membrane microdomain, ii) the CD4 receptor, and the HIV-1 surface envelope glycoprotein gp120. The kinetics of the molecular interactions occuring in this fusion complex were measured with a microtensiometer. This experimental design would allow the evaluation of potential fusion inhibitors such as synthetic analogs of glycosphingolipids.

Keywords : virus, HIV, AIDS, lipids, fusion, membrane, infection. 


\section{ARTICLE}

\section{Introduction}

Le VIH est un virus enveloppé à ARN appartenant à la famille des rétrovirus. Pour qu'une cellule soit infectée par le $\mathrm{VIH}$, il faut que la nucléocapside virale pénètre à l'intérieur de la cellule. Cette opération est assurée par un mécanisme complexe faisant intervenir au moins deux étapes : l'adhésion du virus à la membrane plasmique de la cellule cible et la fusion de l'enveloppe virale avec cette membrane plasmique. Les glycosphingolipides cellulaires organisés en micro-domaines membranaires participent activement à ce processus. Dans cet article, nous décrivons les principales expériences ayant contribué à définir la fonction de ces micro-domaines de glycolipides dans les étapes précoces du cycle d'infection du VIH. Nous proposons un modèle général de fusion viruscellule qui prend en compte le rôle des glycosphingolipides et leur organisation en micro-domaines de la membrane plasmique.

\section{Organisation des glycosphingolipides dans la membrane plasmique}

Les glycosphingolipides sont des composés minoritaires de la membrane plasmique des cellules eucaryotes et procaryotes. Ils sont dérivés d'une structure de base, le céramide, qui comprend une unité sphingosine $\mathrm{N}$-acylée par un acide gras. La partie saccharidique est liée au céramide par une liaison beta1-glycosidique. Les glycosphingolipides sont généralement présents au niveau du feuillet externe de la bicouche lipidique avec lequel ils interagissent via le céramide [1]. Les résidus oligosaccharidiques émergent de la membrane plasmique et recouvrent partiellement la surface des cellules. Cette localisation privilégiée à l'interface cellule-milieu extérieur explique le rôle essentiel joué par les glycosphingolipides dans les communications intercellulaires et les mécanismes d'adhésion cellulaire. Ainsi, il est aujourd'hui bien établi que les glycosphingolipides participent à la régulation de la prolifération et de la différenciation cellulaires. Ils sont impliqués dans certaines voies de transduction de signal et ils assurent la fonction de récepteurs cellulaires pour de nombreux agents pathogènes (bactéries, virus, parasites) et différentes toxines bactériennes [2,3].

En 1985, Thompson et Tillack ont avancé l'hypothèse d'un regroupement sélectif des molécules de sphingolipides dans des zones privilégiées de la membrane plasmique [1]. Cette interprétation était fondée sur des expériences de localisation des glycosphingolipides à la surface de liposomes, à l'aide de ligands spécifiques tels que la toxine cholérique ou certaines lectines. Ces travaux ont permis de proposer un modèle rendant compte de l'organisation des glycosphingolipides dans le feuillet externe de la membrane plasmique (figure 1A). Par la suite, le modèle a été amélioré pour tenir compte du rôle du cholestérol dans l'architecture de ces micro-domaines (figure 1B). Dans ce dernier modèle, les sphingolipides interagissent avec des molécules de cholestérol pour former des microdomaines compacts s'isolant des régions membranaires riches en phosphatidylcholine [4-6]. La fluidité de ces micro-domaines est plus faible que celle des régions environnantes où la phosphatidylcholine est prédominante. Cette différence de fluidité repose sur le mode d'interaction du cholestérol avec les sphingolipides et la phosphatidylcholine, mais aussi sur la structure des chaînes acylées dans ces deux types de lipides. Dans les micro-domaines, le cholestérol vient s'intercaler entre deux molécules adjacentes de sphingolipides qui, du fait de la présence d'une partie polaire volumineuse, laissent un vide dans la partie hydrophobe du feuillet externe de la membrane plasmique. Sa principale fonction serait donc d'être un espaceur moléculaire [5]. 


\section{Bases physico-chimiques de l'organisation des glycosphingolipides en micro-domaines}

Les micro-domaines ou rafts (radeaux) de glycosphingolipides ne sont pas seulement retrouvés au niveau de la membrane des liposomes. On peut les mettre en évidence dans les membranes plasmiques car ils sont insolubles dans le détergent Triton X-100 à $4{ }^{\circ} \mathrm{C}$ [7]. Du fait de leur forte concentration en lipides, ces micro-domaines insolubles dans le détergent et enrichis en glycolipides sont séparés par centrifugation en gradient de saccharose et se retrouvent dans les fractions de faible densité flottant au sommet du gradient. II apparaît donc que les interactions glycolipideglycolipide sont suffisamment fortes pour résister à l'action du détergent. Ces interactions font intervenir les parties apolaires des glycosphingolipides, mais aussi leur partie saccharidique. Afin de mieux comprendre les mécanismes permettant d'exclure les glycosphingolipides des glycérophospholipides tels que la phosphatidylcholine, il est intéressant de comparer les structures de ces différents lipides (figure 2).

Si l'on considère la partie hydrophobe de ces molécules, nous voyons que la chaîne hydrocarbonée de la sphingosine est équivalente sur le plan structural au squelette de glycérol associé à la chaîne acylée en position 1 de la phosphatidylcholine. Ainsi, il suffirait d'une chaîne acylée composée de 14 atomes de carbone pour obtenir une structure équivalente à la sphingosine, c'est-à-dire 18 atomes de carbone et une double liaison 4,5 en configuration trans [6]. Cependant, les chaînes acylées retrouvées dans les phosphoglycérides membranaires en position 1 sont généralement des chaînes saturées à 16 ou 18 atomes de carbone.

Dans la partie céramide des glycosphingolipides, le groupement amine porté par l'atome de carbone $n^{\circ} 2$ de la sphingosine se lie à une chaîne acylée par une liaison amide. Cette chaîne acylée est en général longue (plus de 18 atomes de carbone) et saturée. Par exemple, on retrouve des chaînes à 24 atomes de carbone dans les cérébrosides (GlcCer et GalCer) du cerveau de bœuf, même si des chaînes plus courtes (18:0) sont fréquentes dans la sphingomyéline et les gangliosides de ce même tissu. La prédominance des chaînes saturées contribue ainsi à la haute température de fusion caractéristique des sphingolipides. En revanche, les phosphoglycérides membranaires possèdent une chaine acylée polyinsaturée en position 2 du glycérol. Néanmoins, les chaînes acylées des glycosphingolipides et des phosphoglycérides (en position 2) présentent une analogie de conformation au départ de la chaîne (c'est-à-dire au niveau des 8 premiers atomes de carbone).

Si l'on considère maintenant la région de liaison entre les parties apolaire et polaire de ces lipides, nous voyons que la caractéristique essentielle des sphingolipides est la présence d'un groupement hydroxyle sur l'atome de carbone $\mathrm{n}^{\circ} 3$ de la sphingosine. En outre, la chaîne d'acide gras des sphingolipides peut être alpha-hydroxylée au niveau de l'atome de carbone $n^{\circ} 2$ (en alpha du carbonyle). Les sphingolipides possèdent donc dans cette région deux groupements hydroxyle donneurs de liaison hydrogène et un groupement carbonyle accepteur, alors que les phosphoglycérides ont deux groupements carbonyle accepteurs et aucun groupement donneur.

Les parties polaires des sphingolipides et des phosphoglycérides présentent également une grande diversité. Si l'on excepte la sphingomyéline qui possède un groupement phosphorylcholine identique à celui porté par la phosphatidylcholine, tous les autres sphingolipides possèdent des résidus de sucres leur conférant des propriétés particulières. Les groupements hydroxyle de ces sucres donnent de nombreuses possibilités d'associations intra et/ou intermoléculaires. Ces interactions sucre-sucre contribuent au regroupement des glycosphingolipides en micro-domaines, et à la stabilisation de ces 
complexes. La diversité de la partie saccharidique des glycosphingolipides, comparée au faible nombre de structures polaires des glycérophospholipides, contribue à la flexibilité des modes d'interactions glycolipide-glycolipide et glycolipide-cholestérol dans les micro-domaines membranaires. Elle rend également compte des phénomènes d'adhésion cellule-cellule impliquant un couple de glycosphingolipides (par exemple GM3-Gg3) [4].

\section{Protéines associées aux micro-domaines de glycosphingolipides}

Certaines protéines peuvent être sélectivement associées aux micro-domaines de glycosphingolipides. Ces protéines sont des protéines transmembranaires [8, 9], des protéines ancrées par l'intermédiaire d'un groupement glycosyl-phosphatidylinositol ou GPI [7] ou encore des tyrosine-kinases de la famille Src caractérisées par la présence de deux chaînes acylées facilitant l'interaction avec la membrane [10] (figure 3). L'association de ces protéines aux micro-domaines de sphingolipides et de cholestérol est alors mise en évidence par détection des protéines dans la fraction insoluble au Triton X-100. En ce qui concerne les protéines transmembranaires, les travaux de Sorice et al. [8] montrent que la glycoprotéine CD4, qui est le récepteur du virus de l'immunodéficience humaine $(\mathrm{VIH})$ à la surface des lymphocytes et des macrophages [11], est colocalisée dans des micro-domaines enrichis en GM3. L'immunoprécipitation de CD4 dans la fraction insoluble au Triton X-100 s'accompagne de la copurification du ganglioside GM3, à l'exclusion de tout autre glycosphingolipide. Ces résultats suggèrent une association spécifique de CD4 avec ce ganglioside, bien que l'interaction physique entre CD4 et le GM3 n'ait pas été étudiée par Sorice et al. [8].

\section{Mécanismes moléculaires de la fusion du VIH-1}

L'entrée du VIH de type 1 (VIH-1) dans la cellule nécessite une interaction séquentielle de la glycoprotéine virale de surface, la gp120, tout d'abord avec le récepteur CD4 puis avec un cofacteur de fusion. La fixation du VIH-1 sur le récepteur CD4 entraîne un changement conformationnel de la gp120 qui démasque alors une région particulière, la boucle V3. Cette boucle V3 est un domaine hypervariable de la gp120 qui joue un rôle essentiel dans la fusion virus-cellule [12, 13]. Sa séquence détermine en grande partie la capacité du virus à infecter différentes cibles cellulaires telles que les lymphocytes $\mathrm{CD}^{+}$, les macrophages ou les lignées lymphocytaires $\mathrm{T}$ (cette propriété est appelée tropisme). Au niveau moléculaire, la région de la gp120 comprenant la boucle V3 interagit avec un cofacteur de fusion exprimé à la surface de la cellule cible [14]. Un complexe trimoléculaire se forme, comprenant CD4, la gp120 et le cofacteur (figure 4). Cette étape est nécessaire pour permettre l'interaction du peptide de fusion (extrémité N-terminale de la gp41) avec la membrane plasmique de la cellule cible [15]. La nature du cofacteur (ou corécepteur) peut varier suivant le tropisme du VIH et la nature de la cellule cible, mais les deux principaux sont les récepteurs des chimiokines CXCR4 et CCR5 [16]. CXCR4 interagit avec les isolats T-lymphotropes du VIH (capables d'infecter les lymphocytes $\mathrm{CD} 4^{+}$et les lignées $\mathrm{T}$ transformées, mais non les macrophages), tandis que CCR5 permet l'entrée des isolats primaires M-monocytotropes (infectant les lymphocytes $\mathrm{CD}^{+}$et les macrophages, mais non les lignées T). Pour plus de commodité, on classe aujourd'hui les virus en fonction de la nature du corécepteur utilisé : les virus X4 utilisent CXCR4, tandis que les virus R5 utilisent CCR5 [17]. Les ligands respectifs de ces corécepteurs, c'est dire l'alpha-chimiokine SDF-1 pour CXCR4 et la beta-chimiokine RANTES pour CCR5, inhibent sélectivement la fusion induite par I'une ou l'autre des deux catégories de VIH-1. Les données cristallographiques obtenues récemment 
ont confirmé l'implication de la boucle V3, en association avec les domaines V1 et V2, dans l'interaction gp120-corécepteur [18]. II est important de noter que, en absence de la gp120, les corécepteurs ne sont pas liés à CD4 [19].

Peu avant la découverte du rôle des récepteurs de chimiokines dans le processus de fusion du VIH, deux équipes avaient montré qu'il existait, dans la membrane des globules rouges humains, un facteur de complémentation permettant la fusion du VIH-1 avec des cellules de rongeurs transfectées exprimant le CD4 humain [20-22]. Lorsque les corécepteurs du VIH ont été découverts, on a naturellement pensé qu'un récepteur de ce type était exprimé par les globules rouges. Cependant, le seul récepteur de chimiokine présent sur ces cellules est l'antigène Duffy, lequel s'est avéré inapte à promouvoir la fusion du VIH [23]. En outre, le facteur de fusion érythrocytaire est insensible aux protéases et il n'est pas inactivé par la chaleur. Ces résultats suggéraient ainsi que le cofacteur érythrocytaire pouvait être un lipide membranaire, et non une protéine [24].

\section{Reconstitution in vitro d'un complexe de fusion fonctionnel}

Afin d'étudier les interactions gp120-glycosphingolipides, nous avons utilisé la technique des films monomoléculaires. Le glycosphingolipide purifié est préparé à l'interface eau-air de façon à reconstituer un micro-domaine de glycosphingolipide membranaire [25]. Dans ce système expérimental, l'interaction gp120-glycosphingolipides se traduit alors par une augmentation de la pression de surface (deltapi) à l'interface eau-air à l'aide d'une micro-balance de Langmuir (microtensiomètre muTrough, Kibron Inc., Finlande). Cette méthodologie nous a permis d'identifier deux glycosphingolipides érythrocytaires capables d'interagir avec la gp120. II s'agit du monosialoganglioside GM3 (NeuAcalpha2-3Galbeta1-4Glcbeta1-1Cer) et du globotriaosylcéramide Gb3 (Galalpha1-4Galbeta1-4Glcbeta1-1Cer). L'originalité de ces récepteurs glycolipidiques est qu'ils reconnaissent à la fois CD4 et la gp120 [26, 27]. L'interaction de CD4 avec la monocouche de GM3 se traduit par une augmentation de la pression de surface (figure 5A). L'addition de gp120 sur ce complexe CD4-GM3 provoque une seconde augmentation de pression se traduisant par une rupture de pente au niveau des enregistrements (figure 5A). En reconstituant un micro-domaine membranaire de GM3 sous la forme d'un film monomoléculaire, nous avons donc mis en évidence une interaction séquentielle de GM3 avec CD4, puis avec la gp120. La fixation de la gp120 sur CD4 induit, comme nous l'avons vu plus haut, un changement conformationnel provoquant le démasquage de la boucle V3. Cette boucle V3 peut alors à son tour interagir avec le film monomoléculaire de GM3. En présence d'anticorps anti-V3, l'interaction secondaire boucle V3-GM3 n'a pas lieu (figure 5B). Par conséquent, le micro-domaine de GM3 se comporte comme un site de fixation de la boucle V3 préalablement démasquée à la suite de l'interaction gp120-CD4. L'action de CD4 consiste donc à modifier l'accessibilité de la boucle V3, de telle sorte que l'affinité de ce site pour GM3 augmente. Ainsi, la gp120 est capable d'interagir avec GM3 en absence de CD4, mais à des concentrations plus importantes (figure 6). Cette interaction de faible affinité a pu être mise en évidence pour la gp120 de différents isolats du VIH-1 ainsi que pour la glycoprotéine de surface du $\mathrm{VIH}-2$ (gp105). Des résultats similaires ont été obtenus avec une monocouche de Gb3, en accord avec une étude récente montrant que Gb3 est un cofacteur nécessaire à la fusion du VIH-1 avec les cellules $\mathrm{CD4}^{+}$[28]. Dans ce contexte, nous avons établi l'existence d'une spécificité de la gp120 des isolats $\mathrm{X} 4 \mathrm{du} \mathrm{VIH}-1$ pour $\mathrm{Gb} 3$, tandis que la gp120 des isolats R5 interagit préférentiellement avec GM3 [27]. 


\section{Un modèle de fusion pour le VIH}

Dans notre système expérimental, le glycolipide interagit tour à tour avec CD4, puis avec la gp120. On sait aujourd'hui que le rôle majeur des corécepteurs du VIH (CXCR4 et CCR5) est de favoriser l'attachement de la boucle V3 sur la cellule cible, ce qui a pour effet de déclencher la fusion en libérant la glycoprotéine transmembranaire gp41. Grâce à son extrémité $\mathrm{N}$-terminale très hydrophobe, la gp41 peut alors amorcer le processus de fusion en pénétrant à l'intérieur de la membrane plasmique de la cellule cible. Conceptuellement, un micro-domaine de glycolipide pourrait donc jouer le même rôle que CXCR4 ou CCR5. La localisation de CD4 au niveau de ce microdomaine autorise en effet l'interaction secondaire de la boucle V3 avec d'autres molécules de glycolipides, aboutissant à la formation d'un complexe trimoléculaire CD4-gp120-glycolipide. Un exemple de cette association est donné dans la figure 7 pour un micro-domaine de GM3 et la gp120. Dans ce modèle, la gp120 vient tout d'abord se fixer sur le récepteur CD4 associé à un microdomaine glycolipidique. II s'ensuit un changement conformationnel qui démasque la boucle V3 qui vient alors interagir avec les têtes polaires des molécules adjacentes de glycolipide. Le complexe se déplace à la surface de la cellule, car les micro-domaines de glycolipides sont des éléments constitutifs du feuillet externe de la membrane plasmique. La migration se poursuit jusqu'à ce que la boucle V3 rencontre un récepteur de plus forte affinité que le glycolipide, c'est-à-dire un corécepteur (CCR5 ou CXCR4). Le corécepteur fixe alors la boucle V3, induisant le second changement conformationnel démasquant la gp41 et son peptide de fusion. En absence de corécepteur, cet événement pourrait éventuellement se produire de manière occasionnelle au niveau du microdomaine. Ceci expliquerait l'activité des glycolipides de globules rouges humains (principalement Gb3) dans un système expérimental de fusion ne comportant pas de récepteurs de chimiokines capables de promouvoir la fusion CD4-dépendante du VIH-1. Quoi qu'il en soit, nos résultats montrent l'implication des glycolipides membranaires de la cellule cible dans la formation du complexe de fusion du VIH-1. Notre modèle permet également de rendre compte de l'implication des glycosphingolipides dans l'interaction de divers virus à enveloppe (tels que le virus de la grippe) avec la membrane plasmique des cellules cibles. La mise au point d'analogues synthétiques de glycolipides capables de déstabiliser les interactions virus-glycosphingolipides pourrait représenter une alternative intéressante de thérapeutique antivirale [29].

\section{CONCLUSION}

Ce travail a été financé par la Fondation pour la recherche médicale (Sidaction), I'Institut universitaire de France et le Conseil régional Provence-Alpes-Côte d'Azur.

\section{REFERENCES}

1. THOMPSON TE, TILLACK TW (1985). Organization of glycosphingolipids in bilayers and plasma membranes of mammalian cells. Ann Rev Biophys Chem, 14 : 361-86.

2. HAKOMORI SI (1981). Glycosphingolipids in cellular interaction, differentiation, and oncogenesis. Ann Rev Biochem, 50 : 733-64.

3. KARLSSON KA (1989). Animal glycosphingolipids as membrane attachment sites for bacteria. Ann Rev Biochem, 58 : 309-50. 
4. HAKOMORI SI, IGARASHI Y (1995). Functional role of glycosphingolipids in cell recognition and signaling. J Biochem, $118:$ 1091-103.

5. SIMONS K, IKONEN E (1997). Functional rafts in cell membranes. Nature, 387 : 569-72.

6. BROWN RE (1998). Sphingolipid organisation in biomembranes : what physical studies of model membranes reveal. J Cell Sci, 111 : 1-9.

7. BROWN RE, ROSE J (1992). Sorting of GPI-anchored proteins to glycolipid-enriched membrane subdomains during transport to the apical cell surface. Cell, $68: 533-44$.

8. SORICE M, PAROLINI I, SANSOLINI T, et al. (1997). Evidence for the existence of gangliosideenriched plasma membrane domains in human peripheral lymphocytes. J Lipid Res, 38 : 969-80.

9. SCHEIFFELE P, ROTH MG, SIMONS K (1997). Interaction of influenza virus haemagglutinin with sphingolipid-cholesterol membrane domains via its transmembrane domain. EMBO J, 16 : 5501-8.

10. CASEY PJ (1995). Protein lipidation in cell signalling. Science, $268: 221-5$.

11. BOUR S, GELEZIUNAS R, WAINBERG MA (1995). The human immunodeficiency virus type 1 (HIV-

1) CD4 receptor and its central role in promotion of HIV-1 infection. Microbiol Rev, 59 : 63-93.

12. MOORE JP, NARA PL (1991). The role of the V3 loop of gp120 in HIV infection. AIDS, 5 : S21-33.

13. MOORE JP, BRADFORD A, JAMESON A, WEISS RA, SATTENTAU QJ (1993). The HIV-cell fusion reaction. In : BENTZ J, ed. Viral Fusion Mechanisms. Boca Raton : CRC Press, Inc, 233-89.

14. DIMITROV DS (1997). How do viruses enter cells ? The HIV coreceptors teach us a lesson of complexity. Cell, $91:$ 721-30.

15. BINLEY J, MOORE JP (1997). The viral mousetrap. Nature, 387 : 346-8.

16. CLAPHAM PR, WEISS RA (1997). Spoilt for choice of coreceptors. Nature, 388 : 230-1.

17. BERGER EA, DOMS RW, FENYÖ EM, et al. (1998). A new classification for HIV-1. Nature, 391 : 240.

18. KWONG PD, WYATT R, ROBINSON J, SWEET RW, SODROWSKI J, HENDRICKSON WA (1998). Structure of an HIV gp120 envelope glycoprotein in complex with the CD4 receptor and a neutralizing human antibody. Nature, $393: 648-59$.

19. LAPHAM CL, OUYANG J, CHANDRASEKHAR B, NGUYEN NY, DIMITROV DS, GOLDING H (1996). Evidence for cell surface association between fusin and the CD4-gp120 complex in human cell lines. Science, $274: 602-5$.

20. DRAGIC T, PICARD L, ALIZON M (1995). Proteinase-resistant factors in human erythrocyte membranes mediate CD4-dependant fusion with cells expressing human immunodeficiency virus type 1 envelope glycoproteins. J Virol, 69 : 1013-8. 
21. PICARD L, DRAGIC T, WIELS J, ALIZON M (1996). Possible role of nonprotein components of the plasma membrane in CD4-dependent membrane fusion and HIV entry. Perspect. Drug Discov Design, $5: 143-53$.

22. PURI A, MORRIS SJ, JONES P, RYAN M, BLUMENTHAL R (1996). Heat-resistant factors in human erythrocyte membrane mediate CD4-dependent fusion with cells expressing HIV-1 envelope glycoproteins. Virology, $219: 262-7$.

23. DORANZ BJ, RUCKER J, YI Y, et al. (1996). A dual-tropic primary HIV-1 isolate that uses fusin and beta-chemokine receptors CKR-5, CKR-3, and CKR-2b as fusion cofactors. Cell, 85 : 1149-58.

24. PURI A, HUG P, MUNOZ-BARROSO I, BLUMENTHAL R (1998). Human erythrocyte glycolipids promote HIV-1 envelope glycoprotein-mediated fusion of $\mathrm{CD} 4^{+}$cells. Biochem Biophys Res Commun, $242: 219-25$.

25. HAMMACHE D, PIÉRONI G, YAHI N, et al. (1998). Specific interaction of HIV-1 and HIV-2 surface envelope glycoproteins whith monolayers of galactosylceramide and ganglioside GM3. J Biol Chem, 273 : 7967-71.

26. HAMMACHE D, YAHI N, PIERONI G, ARIASI F, TAMALET C, FANTINI J (1998). Sequential interaction of CD4 and HIV-1 gp120 with a reconstituted membrane patch of ganglioside GM3 : implications for the role of glycolipids as potential HIV-1 fusion cofactors. Biochem Biophys Res Commun, 246 : 11722.

27. HAMMACHE D, YAHI N, MARESCA M, PIERONI G, FANTINI J (1999). Human erythrocyte glycosphingolipids as alternative cofactors for human immunodeficiency virus type 1 (HIV-1) entry : evidence for CD4-induced interactions between HIV-1 gp120 and reconstituted membrane microdomains of glycosphingolipids (Gb3 and GM3). J Virol, $73: 5244-8$.

28. PURI A, HUG P, JERNIGAN K, et al. (1998). The neutral glycosphingolipid globotriaosylceramide promotes fusion mediated by a CD4-dependent CXCR4-utilizing HIV type 1 envelope glycoprotein. Proc Natl Acad Sci USA, 98 : 14435-40.

29. FANTINI J (2000). Synthetic soluble analogs of glycolipids for strudies of virus-glycolipid interactions. Meth Enzymol, $311: 626-38$. 
Illustrations
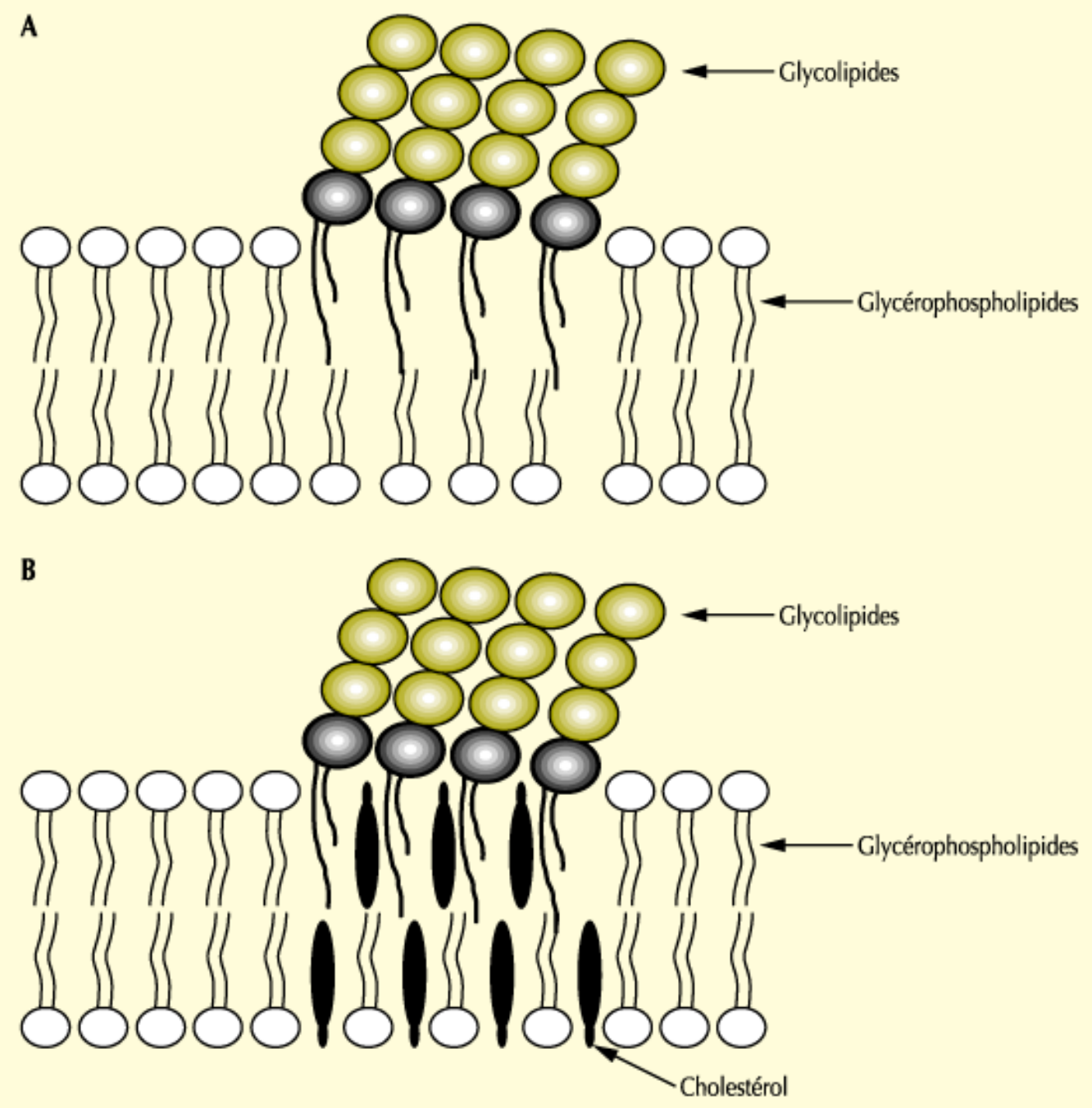

Figure 1. Schéma de l'organisation membranaire des glycosphingolipides. A : d'après Thompson et Tillack [1]. Les glycosphingolipides se regroupent pour former des micro-domaines dans le feuillet externe de la membrane plasmique. Dans ce modèle d'organisation, le rôle du cholestérol dans l'architecture des micro-domaines n'est pas pris en compte. B : d'après Simons et Ikonen [5]. Dans ce modèle, les molécules de glycosphingolipides interagissent avec des molécules de cholestérol pour former des micro-domaines compacts s'isolant des régions riches en phosphatidylcholine. Le rôle du cholestérol dans l'architecture de ces micro-domaines est de s'intercaler entre les molécules de glycosphingolipides (notion d'espaceur moléculaire). 


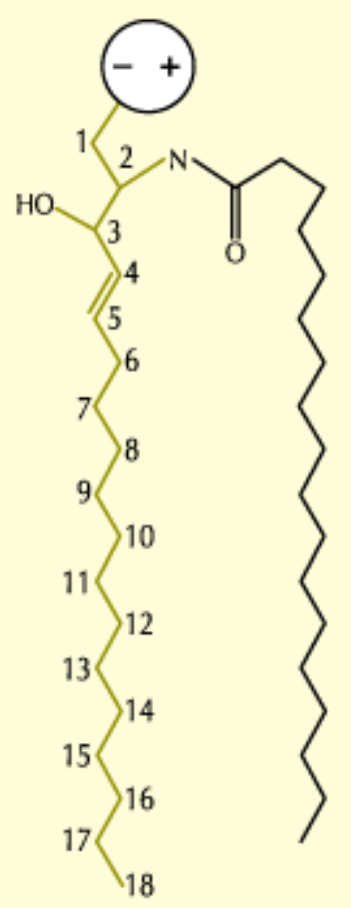

18:0

A

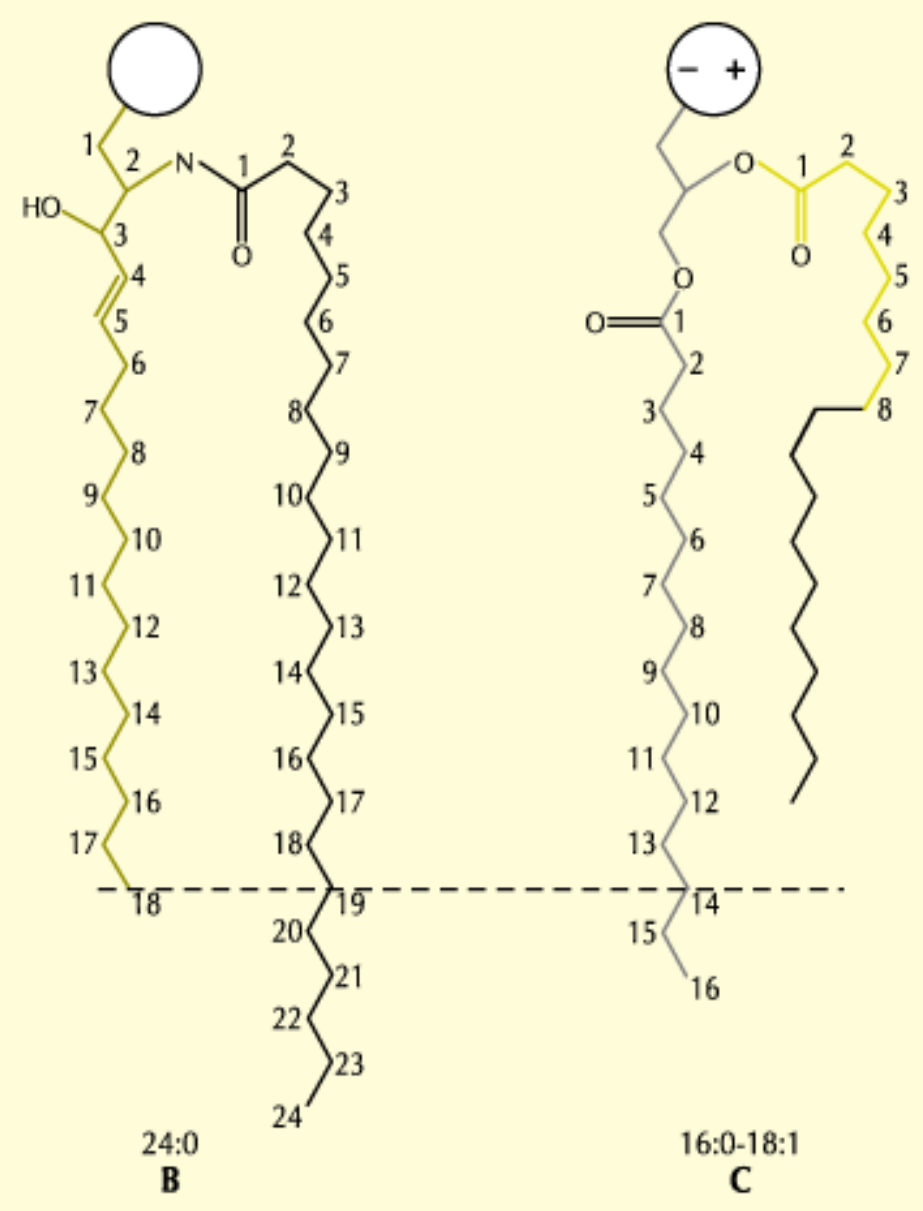

Figure 2. Structure chimique des sphingolipides et de la phosphatidylcholine. $A$ : sphingomyéline. $B$ : glycosphingolipide. C : phosphatidylcholine. La nature des acides gras présents dans ces molécules est indiquée (modifié d'après Brown et Rose [6]). 


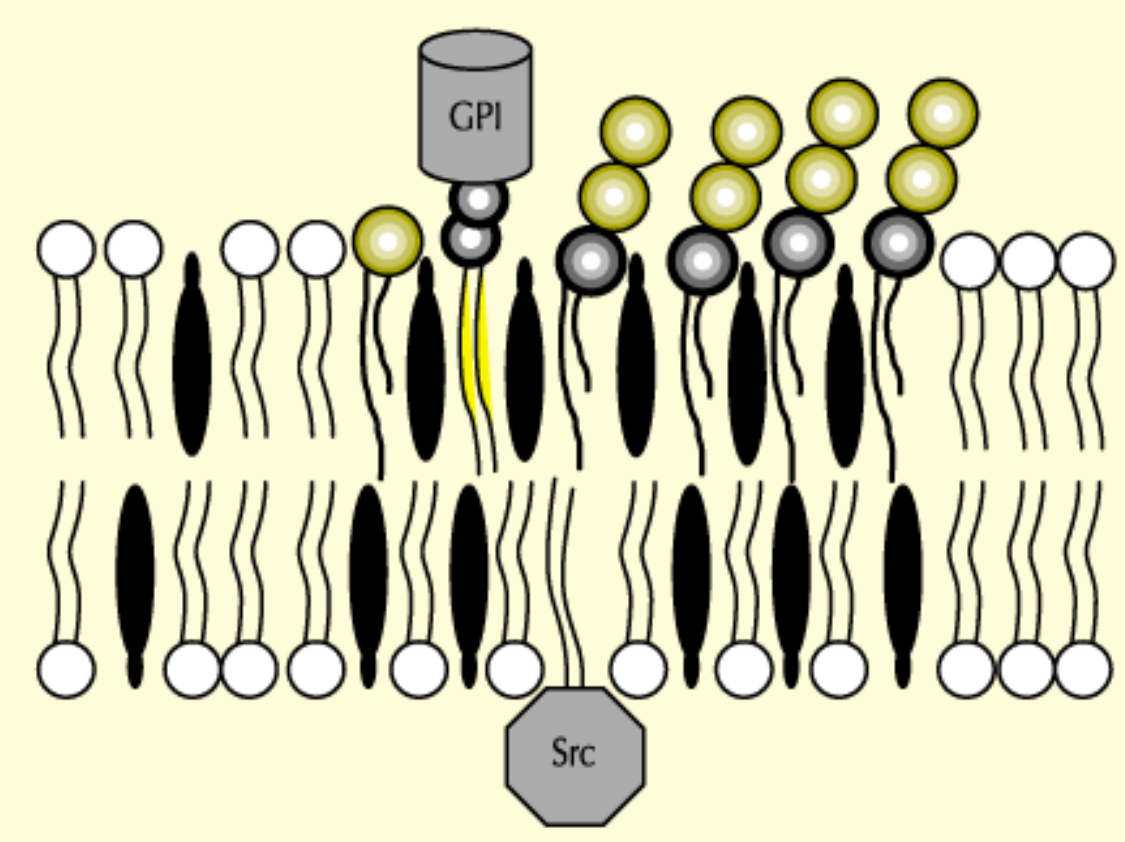

Figure 3. Protéines associées aux micro-domaines glycosphingolipidiques. GPI : protéine ancrée par glycosyl-phosphatidylinositol; Src : tyrosine-kinase de la famille Src ancrée dans la membrane par deux chaînes acylées. 


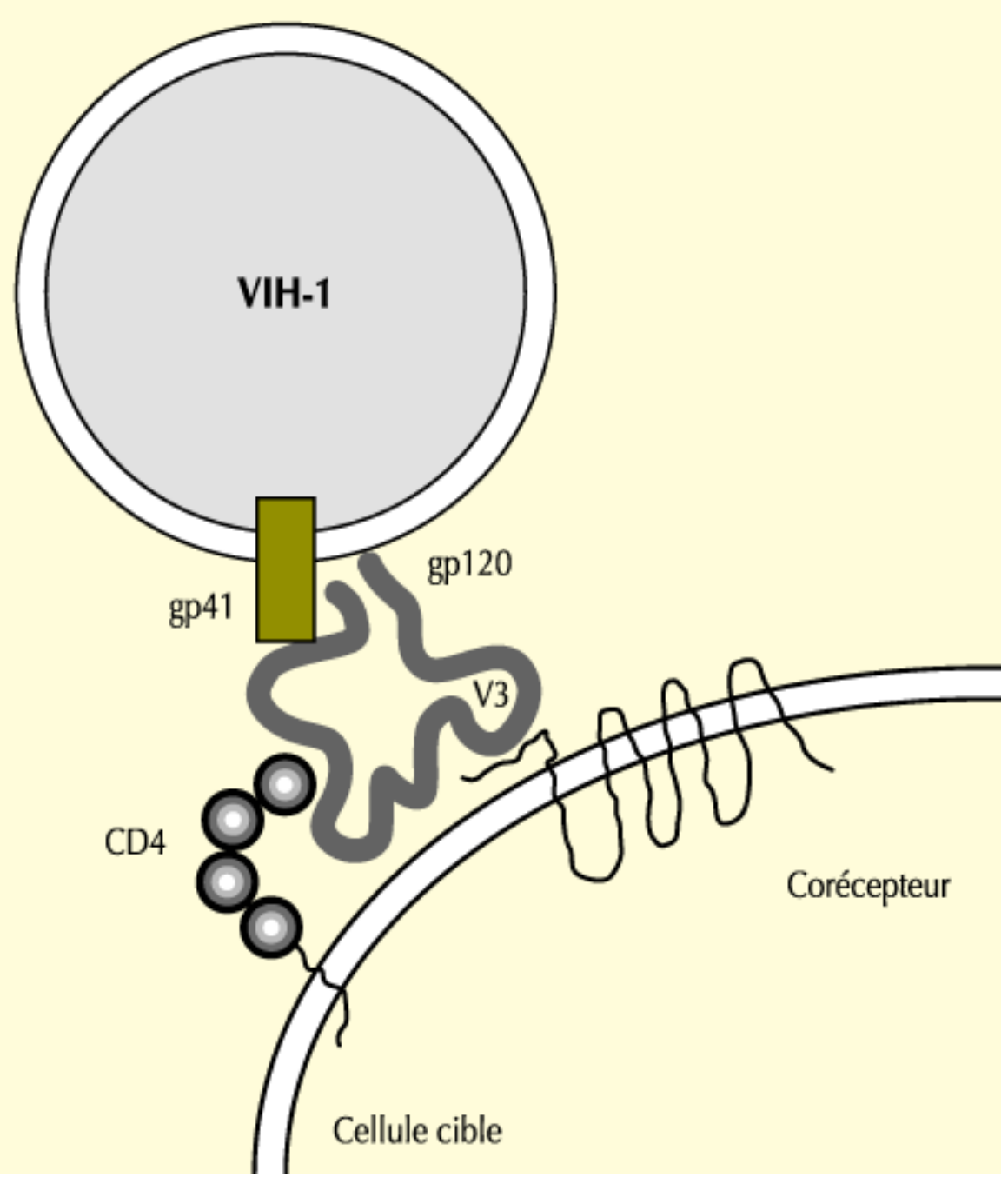

Figure 4. Fusion du VIH-1 avec une cellule $C D 4^{+}$. L'interaction du VIH-1 avec les cellules exprimant le récepteur CD4 se fait en deux temps. La gp120 se lie tout d'abord à CD4, ce qui a pour effet de démasquer sa boucle V3. La boucle V3 devient alors accessible pour sélectionner un corécepteur dont la reconnaissance détermine le tropisme du virus. Ce n'est qu'après avoir formé le complexe trimoléculaire CD4-gp120corécepteur que la fusion peut démarrer sous le contrôle de l'extrémité $\mathrm{N}$-terminale de la gp41. 

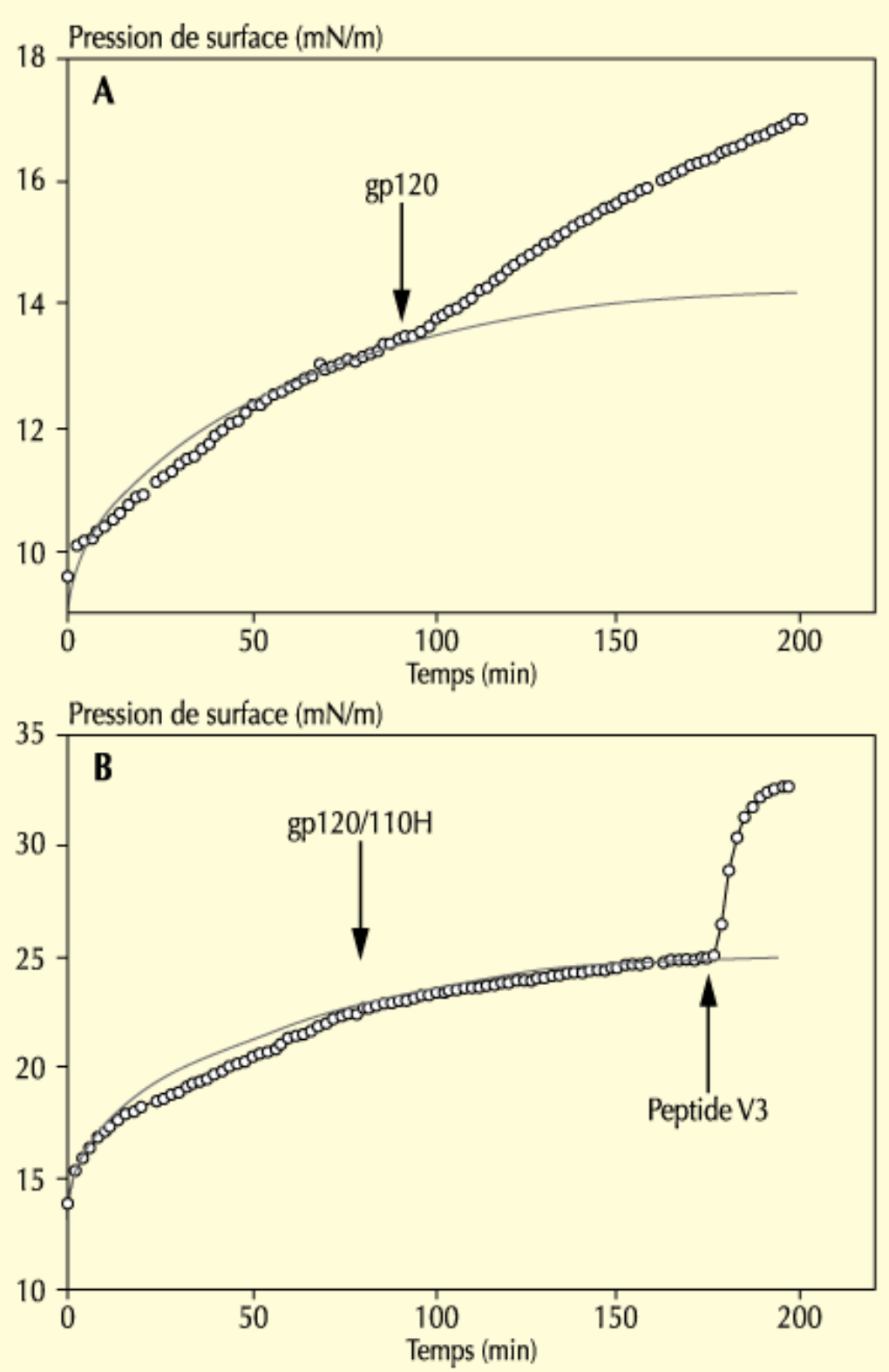

Figure 5. Mise en évidence d'un complexe CD4-GM3gp120. L'addition de CD4 $(0,5 \mathrm{ng} / \mathrm{ml})$ sur un film monomoléculaire de GM3 provoque une augmentation de la pression de surface modélisée par la courbe en trait plein. L'ajout de gp120 (1,85 nM) provoque une augmentation supplémentaire de pression (A) qui est inhibée par l'anticorps monoclonal anti-V3 110-H (B). Dans ce dernier cas, la fonctionnalité de la monocouche de GM3 est controlée en ajoutant un peptide V3 qui n'a pas été préalablement neutralisé par l'anticorps. 


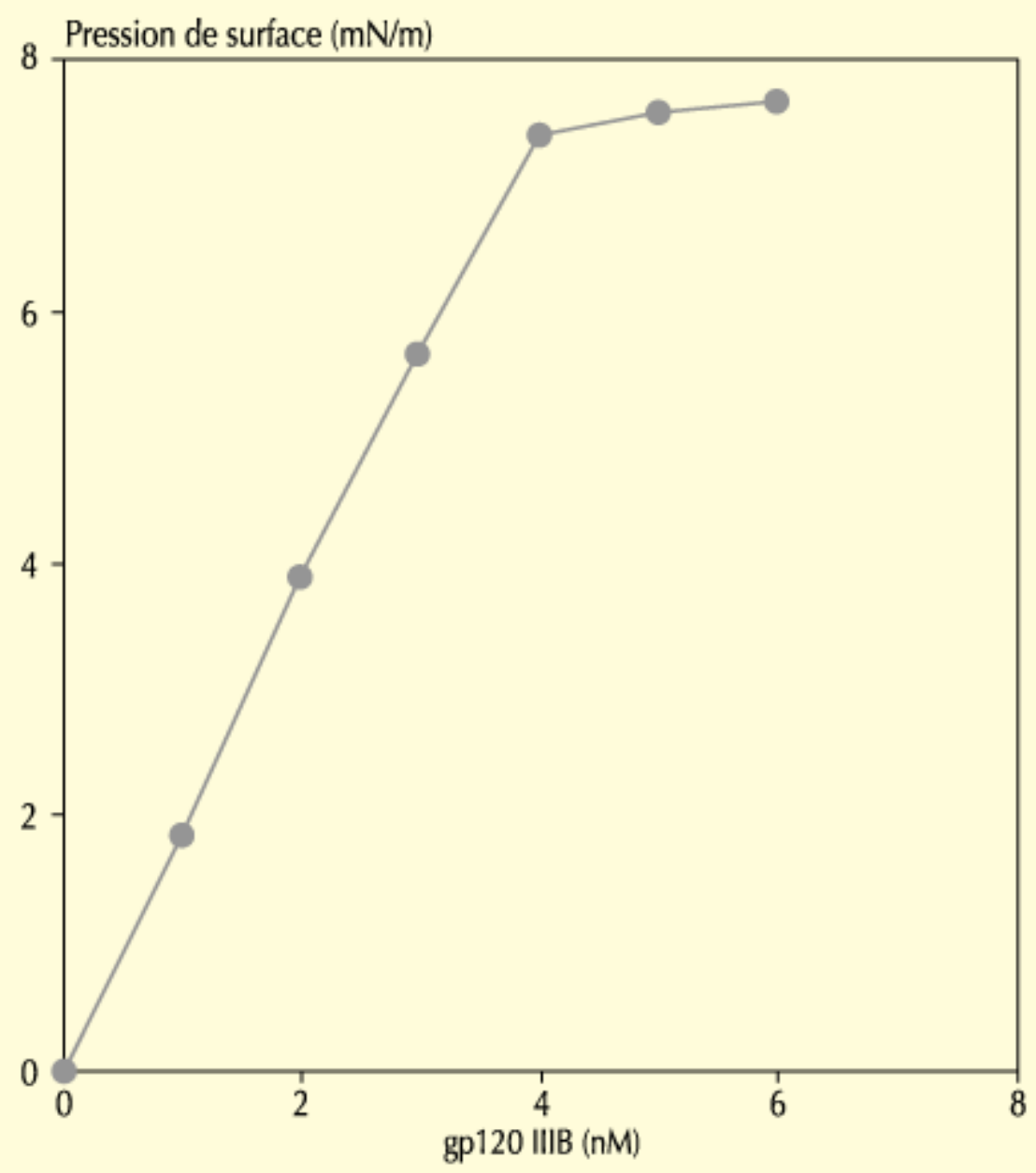

Figure 6. Interaction de la gp120 avec un film monomoléculaire de GM3. Le film monomoléculaire est préparé afin d'avoir une pression de surface initiale de $10 \mathrm{mN} / \mathrm{m}$. Les données montrent une augmentation de la pression de surface induite par l'addition de différentes concentrations de gp120. 


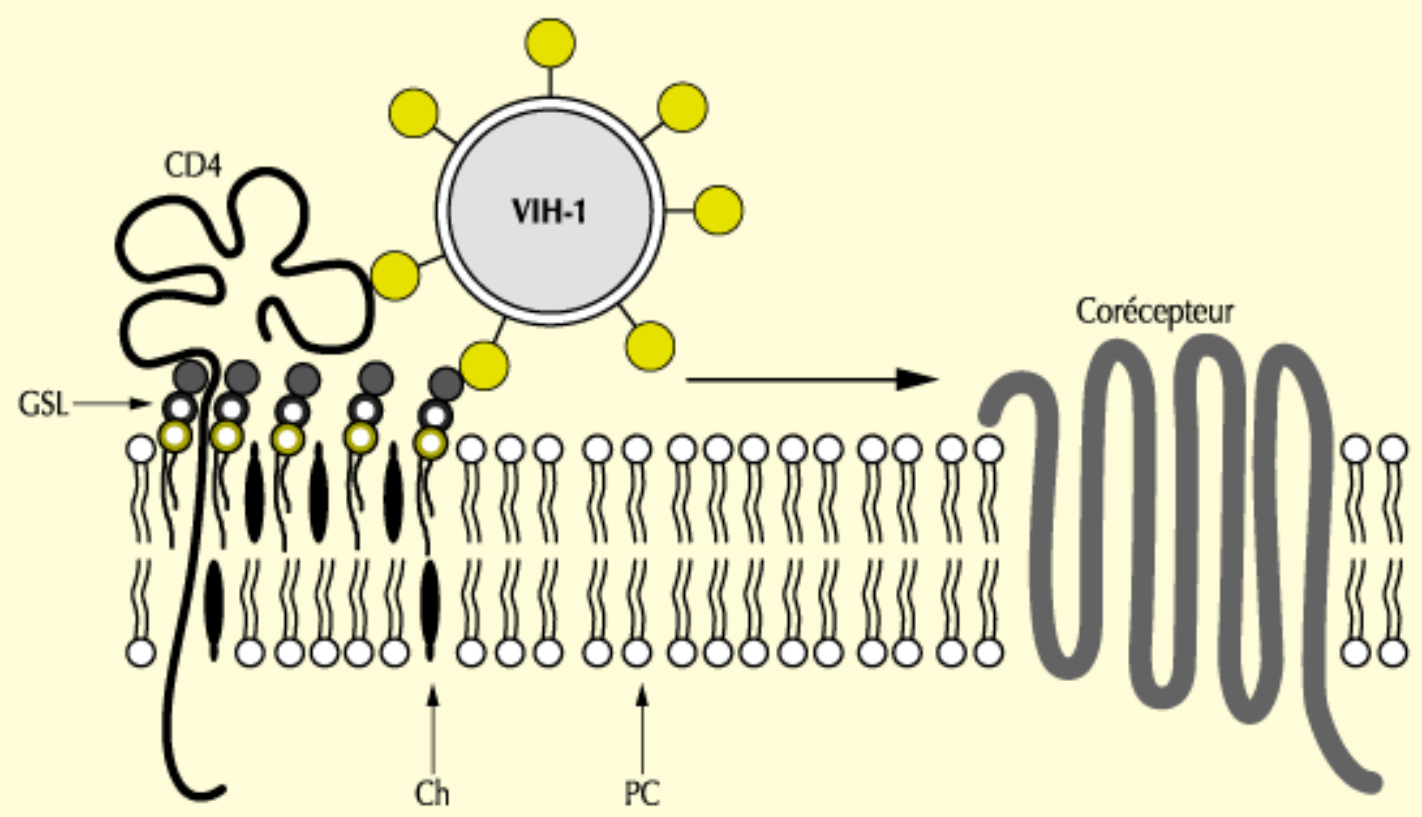

Figure 7. Fusion du VIH-1 au niveau d'un micro-domaine glycolipidique. Au niveau de la membrane plasmique de la cellule cible, CD4 interagit avec un micro-domaine de glycolipides. La fixation de la gp120 sur son récepteur CD4 induit un changement conformationnel qui démasque la boucle V3 qui vient alors interagir avec les têtes polaires des molécules adjacentes de glycolipides. Le complexe se déplace à la surface de la cellule jusqu'à ce que la boucle V3 rencontre un récepteur de plus forte affinité que le glycolipide, c'est-à-dire un corécepteur. Le corécepteur fixe alors la boucle V3, induisant le second changement conformationnel, nécessaire à la libération de l'extrémité $N$-terminale de la gp41 qui va initier le processus de fusion en s'insérant dans la membrane plasmique de la cellule cible. Ch : cholestérol ; GSL : glycosphingolipide; PC : phosphatidylcholine. 